\title{
PsoBest - Das Deutsche Psoriasis-Register: Ein Abbild der Versorgung
}

\author{
PsoBest - The German Psoriasis Registry: An Image of Routine Care
}

Autoren

Institut
S. J. Rustenbach', S. Knopf, Z. Foos, K. Haack, M. El Hamoumi, J. Hoffmann, C. Spehr

Competenzzentrum Versorgungsforschung in der Dermatologie (CVderm), Institut für Versorgungsforschung in der Dermatologie und bei Pflegeberufen (IVDP), Universitätsklinikum Hamburg-Eppendorf, Hamburg

\section{Bibliografie}

DOI http://dx.doi.org/

10.1055/s-0034-1392876

Akt Dermatol 2015; 41: 326-329

(c) Georg Thieme Verlag KG

Stuttgart · New York

ISSN 0340-2541

\section{Korrespondenzadresse:}

Dr. Stephan Jeff Rustenbach

Competenzzentrum

Versorgungsforschung in der

Dermatologie (CVderm)

Institut für Versorgungsforschung

in der Dermatologie und bei

Pflegeberufen (IVDP)

Universitätsklinikum

Hamburg-Eppendorf

Martinistraße 52

20246 Hamburg

s.rustenbach@uke.de

\section{Zusammenfassung \\ $\nabla$}

PsoBest ist heute das größte Patientenregister in der deutschen Dermatologie. Im Auftrag der Deutschen Dermatologischen Gesellschaft und des Berufsverbandes der Deutschen Dermatologen dokumentiert das Competenzentrum Versorgungsforschung in der Dermatologie gemeinsam mit den teilnehmenden Dermatologen und ihren Patienten die Versorgung der mittelschweren und schweren Psoriasis und Psoriasis-Arthritis in Deutschland. Im Rahmen des Europäischen

\section{Einleitung}

Das Patientenregister PsoBest wurde 2008 vom CVderm (Competenzentrum Versorgungsforschung in der Dermatologie) im Auftrag der DDG (Deutschen Dermatologischen Gesellschaft) und des BVDD (Berufsverbandes der Deutschen Dermatologen) am Universitätsklinikum HamburgEppendorf eingerichtet. Ziel des Registers ist die Gewinnung eines umfassenden Abbilds der Versorgung von Patienten mit mittelschwerer und schwerer Psoriasis und Psoriasis-Arthritis in Deutschland.

Ein solches Abbild der Versorgung kann freilich nur in gemeinschaftlicher Zusammenarbeit mit den versorgenden und versorgten Akteuren den Dermatologen und deren Patienten entstehen. Das Abbild wird umso schärfer ausfallen, je höher die Beteiligung ausfällt. Um dieses Ziel zu erreichen, sollten sich also möglichst alle Psoriasis-versorgenden dermatologischen Praxen und Kliniken zur Teilnahme bei PsoBest anmelden und möglichst alle Patienten an das Register melden, die zur Aufnahme in das Register geeignet sind und einer Teilnahme freiwillig, informiert und schriftlich zugestimmt haben. Zurzeit beteiligen sich sowohl Klinikambulanzen als auch niedergelassene Dermatologen aus ganz Deutschland $(\bullet$ Abb. 1).
Netzwerks der Psoriasis-Register trägt PsoBest proaktiv zu einem internationalen Frühwarnund Überwachungssystem der Arzneimittelsicherheit bei. Ziel des Registers ist die Gewinnung eines umfassenden Abbilds der Versorgung. Dank der großen und weiterhin steigenden Anzahl aktiv teilnehmender Dermatologen und Patienten aus ganz Deutschland sowie einer eingespielten Zusammenarbeit und Kommunikation auf allen Studienebenen wird dieses Abbild immer schärfer.

\section{Datenmeldung an PsoBest}

Die Anmeldung als PsoBest-Zentrum kann beispielsweise unkompliziert über die PsoBestHomepage (www.psobest.de) erfolgen. Das CVderm übersendet nachfolgend die notwendigen Studienunterlagen und begleitet danach bei der Durchführung.

Zur Meldung an PsoBest geeignet sind alle volljährigen und teilnahmefähigen Patienten, bei denen eine Psoriasis vulgaris mit oder ohne Gelenkbeteiligung vorliegt, sobald eine systemische Behandlung der Psoriasis begonnen wird, die der Patient noch nie zuvor in seinem Leben erhalten hat (dazu zählen alle eingesetzten konventionellen Systemtherapeutika, Biologika, Biosimilars und "small molecules“).

Nach der Registrierung wird jeder Patient - unabhängig von späteren Therapiewechseln oder -pausen - über 10 Jahre von PsoBest beobachtet, indem regelmäßig Folgevisiten beim Dermatologen dokumentiert werden ( $\bullet$ Abb. $\mathbf{2}$ ).

Die Folgevisiten werden durch zwischenzeitliche Patientenbefragungen durch das CVderm ergänzt. Die Dokumentation der dermatologischen Behandlung und Versorgung erfolgt standardisiert in sog. CRFs (case report forms). Diese umfassen für den Arzt Angaben über aktuelle und vorausgehende Therapien, Begleiterkrankungen und 


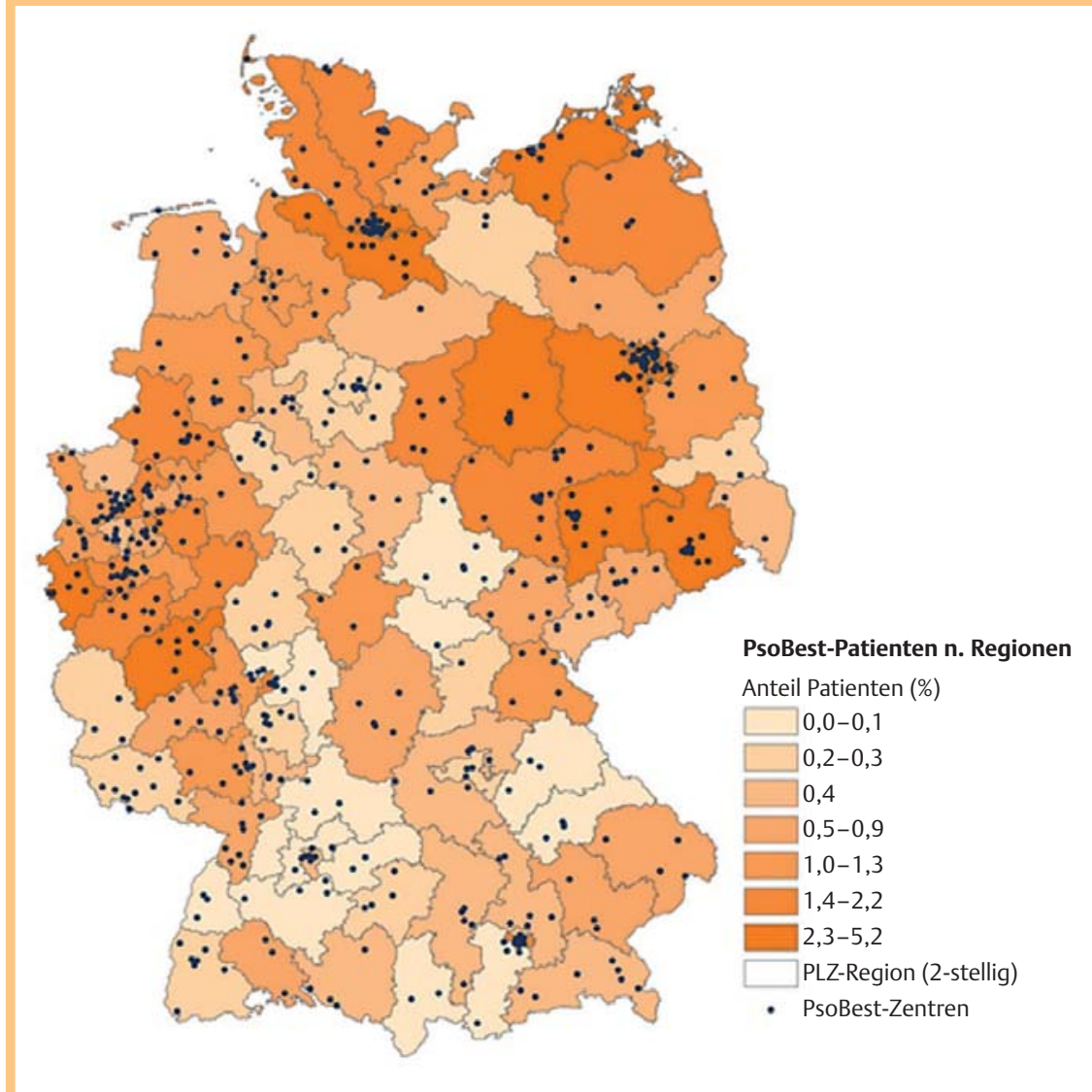

Abb. 1 PsoBest-Partner. Dermatologen und Patienten aus ganz Deutschland beteiligen sich aktiv am Register.

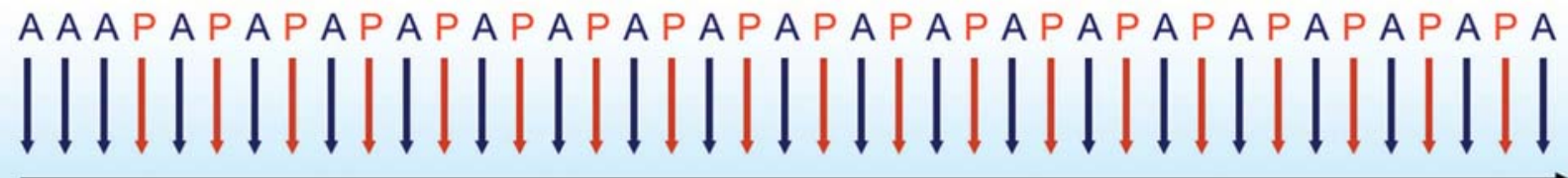

Month $\begin{array}{llllllllllllllllllllllllllllllllllllllllllll}0 & 3 & 6 & 9 & 12 & 15 & 18 & 21 & 24 & 27 & 30 & 33 & 36 & 39 & 42 & 45 & 48 & 51 & 54 & 57 & 60 & 63 & 66 & 69 & 72 & 75 & 78 & 81 & 84 & 87 & 90 & 93 & 96 & 99 & 102 & 105 & 108 & 111 & 114 & 117 & 120\end{array}$

Year 0 1

2

3

4

5

6

7

8

9

10

Abb.2 Dokumentationstermine in PsoBest. Ärzte und Patienten füllen in regelmäßigen Abständen über 10 Jahre Fragebögen aus (A: Visite beim Arzt, P: Patientenbefragung durch CVderm).

-medikationen, zum allgemeinen und dermatologischen Status nebst PASI und ggf. zu Gelenkbeteiligung sowie zu unerwünschten Ereignissen und für den Patienten Angaben über Soziodemografie, Symptomatik, Schweregrad und Gesundheitszustand, zur Lebensqualität und Behandlungszielen und -nutzen sowie zur Versorgungs- und Therapiezufriedenheit. Schwerwiegende unerwünschte Ereignisse werden jederzeit umgehend per Formular gemeldet.

Die eingehenden Dokumentationen unterliegen im CVderm einer kontinuierlichen und standardisierten Qualitätssicherung (inklusive Nachfragemanagement und unterstützenden Monitoringbesuchen) und Weiterverarbeitung. Das Datenschutzkonzept gewährleistet den verantwortungsvollen und sicheren Umgang mit den gemeldeten Personen- und Gesundheitsdaten.

\section{Datenberichte aus PsoBest}

$\nabla$

Der Registerfortschritt wird wochenaktuell auf der PsoBestHomepage berichtet. Zweimal pro Jahr werden dort auch die kumulierenden Behandlungen und Behandlungszeiten aktualisiert ( Tab.1).

Jedes Zentrum erhält zweimal jährlich eine Charakterisierung der Zentrumspatienten und deren Behandlungsverlauf in Relation zu allen anderen Zentren sowie jeweils eine Seite Charakterisierung und Verlauf pro Patient.

Pharmakovigilanzberichte werden in halbjährlichen Abständen angefertigt, wissenschaftlich sowie medizinisch bewertet und an die pharmazeutischen Hersteller versandt.

Aktuelle Sachstände und Neuigkeiten werden quartalsweise über Newsletter und im Internet mitgeteilt. Einmal jährlich erfolgt ein Treffen der PsoBest-Prüfärzte, dort werden zudem aktuelle Daten und Ergebnisse präsentiert. 
Tab. 1 Expositionshäufigkeiten und -zeiten. Für insgesamt 3510 Patienten wurden bis 31.12.2014 über 7000 Behandlungsjahre beobachtet.

\begin{tabular}{|c|c|c|c|c|c|}
\hline & $\begin{array}{l}\text { Behandlung } \\
\text { bei Einschluss } \\
\text { (n) }\end{array}$ & $\begin{array}{l}\text { Behandlungswechsel } \\
\text { auf ... } \\
\text { (n) }\end{array}$ & $\begin{array}{l}\text { Jemals erhalten } \\
\text { in PsoBest } \\
\text { (n) }\end{array}$ & $\begin{array}{l}\text { Behandlungsjahre } \\
\text { (Summe) }\end{array}$ & $\begin{array}{l}\text { Jahre seit } \\
\text { Erstbeginn } \\
\text { (Summe) }\end{array}$ \\
\hline Adalimumab & 414 & 219 & 773 & 1253,4 & 1553,2 \\
\hline Efalizumab & 34 & 7 & 193 & 23,2 & 122,8 \\
\hline Etanercept & 229 & 122 & 567 & 616,4 & 992,1 \\
\hline Infliximab & 82 & 50 & 229 & 246,4 & 363,5 \\
\hline Ustekinumab & 227 & 168 & 395 & 827,6 & 965,5 \\
\hline Golimumab & 22 & 23 & 45 & 86,8 & 113,0 \\
\hline Cyclosporin & 215 & 99 & 718 & 332,7 & 616,7 \\
\hline Fumaderm & 1311 & 89 & 2484 & 1807,0 & 2390,5 \\
\hline MTX & 882 & 322 & 1886 & 1826,7 & 2319,1 \\
\hline
\end{tabular}

Wissenschaftliche Publikationen sind über eine Verfahrensordnung und einen Publikationsplan geregelt, sie erfolgen, sobald genügend Daten für eine jeweilige Fragestellung vorliegen $[1,2]$.

\section{Wissenschaftliche Grundlagen \\ $\nabla$}

Zur Abbildung der Versorgung einer Erkrankung in größeren Regionen und über längere Zeit sind Patientenregister besonders geeignet [3-8]. Die Beobachtung der Versorgung erfolgt unter Alltagsbedingungen ohne in diese einzugreifen und umfasst auch Patienten mit z.B. allen Begleiterkrankungen und -medikationen, wie sie in der Routineversorgung auftreten.

Diese breite Datenbasis wird erstmals robuste Daten zur Wirksamkeit und Sicherheit der systemischen Langzeittherapie der Psoriasis in der Routineversorgung Deutschlands generieren und es ermöglichen, differenzierte Fragestellungen wie beispielsweise zu speziellen Patientengruppen, zum Therapieansprechen und zu Therapieregimen zu klären.

Das methodische Konzept wurde anhand der einschlägigen internationalen Richtlinien entwickelt und in einem Studienprotokoll vorab festgelegt [4-6,9-16]. Es liegt ein positives Votum der Ethikkommission der Landesärztekammer Hamburg (Nr.2805) vor.

Die Konzeption wurde zudem in enger Abstimmung mit dem Rabbit (Rheumatoide Arthritis: Beobachtung der Biologika-Therapie) und Registern des Netzwerks der Europäischen PsoriasisRegister (psonet) vorgenommen, sodass gemeinsame Auswertungen und Datenvergleiche möglich sind $[3,7,8,17,18]$. Im Rahmen des ENCePP-Netzwerks psonet trägt PsoBest proaktiv zu einem internationalen Frühwarn- und Überwachungssystem der Arzneimittelsicherheit bei.

\section{Fazit}

$\nabla$

Das Netzwerk der Europäischen Psoriasis-Register umfasst eine bedeutsame geografische Fläche und eine sehr große Patientenzahl. Potenzielle Signale zur Arzneimittelsicherheit können hier rasch erkannt, überprüft und kommuniziert werden.

Die Güte dieses Überwachungssystems steigt mit der Anzahl der beobachteten Patienten und deren geografischer Verteilung. Dies gilt ebenso auf nationaler Ebene für PsoBest, das jetzt schon größte Patientenregister in der deutschen Dermatologie. Das Gemeinschaftsprojekt fußt auf einem verteilten und breiten Spektrum aktiv teilnehmender Dermatologen und Patienten so- wie einer guten und qualitätsgesicherten Zusammenarbeit und Kommunikation auf allen Studienebenen über Jahre. Die hohe Anzahl der teilnehmenden Dermatologen und ihrer Patienten zeigt, dass dies gelingt.

Dank der aktiven Teilnahme und nachhaltigen Unterstützung der teilnehmenden Dermatologen aus dem niedergelassenen Bereich und aus den Kliniken ist PsoBest bereits heute eine wichtige Datenquelle für die deutsche Dermatologie.

\section{Interessenkonflikt}

$\nabla$

PsoBest wird von folgenden pharmazeutischen Unternehmen finanziell unterstützt: AbbVie, Amgen, Biogen, Celgene, JanssenCilag, medac, Novartis, Pfizer.

\section{Abstract}

\section{PsoBest - The German Psoriasis Registry: An Image of Routine Care}

PsoBest is the largest patient registry in German dermatology to date. Real world routine care in moderate and severe psoriasis and psoriatic arthritis in Germany is observed in cooperation of dermatologists, patients and the German Center for Health Services Research in Dermatology on behalf of the German Dermatologic Society and the Association of German Dermatologists. As part of the European network of psoriasis registries, PsoBest proactively contributes to an international rapid alert and surveillance system for drug safety. Purpose of the registry is to retrieve a comprehensive image of routine care. Due to the large and continuously growing number of actively participating dermatologists and patients from all over Germany, as well as the well-rehearsed cooperation and communication between all levels of the registry, the image is getting shaper.

\section{Literatur}

1 Augustin M, Spehr C, Radtke MA et al. German Psoriasis Registry PsoBest: Objectives, methodology and first baseline data. J Dtsch Dermatol Ges 2014; 12: $48-57$

2 Radtke MA, Rustenbach SJ, Reusch $M$ et al. Influenza vaccination rate among patients with moderate to severe psoriasis. J Dtsch Dermatol Ges 2013; 11: 837-844 
3 Zink A, Listing J, Klindworth C et al. The national database of the German Collaborative Arthritis Centres: I. Structure, aims, and patients. Ann Rheum Dis 2001; 60: 199-206

4 Müller D, Augustin M, Banik $N$ et al. Memorandum Register für die Versorgungsforschung. Gesundheitswesen 2010; 72: 824-839

5 Glicklich R, Dreyer N. Registries for Evaluating Patient Outcomes: A User's Guide. 2nd ed. Rockville (MD): Agency for Healthcare Research and Quality (US); 2010

6 The European Network of Centres for Pharmacoepidemiology and Pharmacovigilance (ENCePP). Guide on Methodological Standards in Pharmacoepidemiology. EMA/95098/2010. http://www.encepp.eu/ standards_and_guidances; letzter Zugriff: 27.3.2015

7 Schmitt-Egenolf $M$. Psoriasis therapy in real life: the need for registries. Dermatology 2006; 213: 327-330

8 Lecluse LL, Naldi L, Stern RS et al. National registries of systemic treatment for psoriasis and the European 'Psonet' initiative. Dermatology 2009; 218: 347-356

9 Agency EM. The Rules Governing Medicinal Products in the European Union - Guidelines on Pharmacovigilance for Medicinal Products for Human Use. 2008; Vol. 9a: http://ec.europa.eu/health/files/pharmacos/docs/doc2005/12-05/draft_of_volume_9a_12_2005_en.pdf; letzter Zugriff: 27.3 .2015

10 U.S. Department of Health and Human Services FDA Center for Drug Evaluation and Research. Guidance for industry: patient-reported outcome measures: use in medical product development to support labeling claims: draft guidance. Health Qual Life Outcomes 2006; 4: 79
11 Agency EM. Note for Guidance on Clinical Safety Data Management. 2009; http://www.ema.europa.eu/docs/en_GB/document_library/ Scientific_guideline/2009/09/WC500002749.pdf; letzter Zugriff: 27.3.2015

12 Medical Dictionary for Regulatory Activities terminology is the international medical terminology developed under the auspices of the International Conference on Harmonization of Technical Requirements for Registration of Pharmaceuticals for Human Use (ICH). http:// www.meddra.msso.com; letzter Zugriff: 27.3.2015

13 (ISPE) ISoP. Guidelines for good pharmacoepidemiology practices (GPP). Pharmacoepidemiol Drug Saf 2008; 17: 200 - 208

14 Leitlinien und Empfehlungen zur Sicherung von Guter Epidemiologischer Praxis (GEP). http://www.gesundheitsforschungbmbf.de/_media/Empfehlungen_GEP.pdf; letzter Zugriff: 27.3.2015

15 Elm E von, Altman DG, Egger $M$ et al. Strengthening the Reporting of Observational Studies in Epidemiology (STROBE) statement: guidelines for reporting observational studies. BMJ 2007; 335: 806-880

16 Guideline on good pharmacovigilance practices (GVP) Module VIII Post-authorisation safety studies. European Medicines Agency and Heads of Medicines Agencies; 2012

17 Ormerod AD, Augustin M, Baker $C$ et al. Challenges for synthesising data in a network of registries for systemic psoriasis therapies. Dermatology 2012; 224: 236-243

18 Garcia-Doval I, Rustenbach SJ, Stern R et al. Psonet Network. Systemic psoriasis therapy shows high between-country variation: a sign of unwarranted variation? Cross-sectional analysis of baseline data from the PSONET registries. Br J Dermatol 2013; 169: 710-714 